\title{
Normatização dos Equipamentos e Técnicas para Realização de Exames de Mapeamento Ambulatorial de Pressão Arterial (MAPA) e de Monitorização Residencial da Pressão Arterial (MRPA)
}

\author{
Coordenador \\ Jorge Ilha Guimarães \\ Editor \\ Marco Antônio Mota Gomes \\ Co-editores \\ Décio Mion Jr e Fernando Nobre \\ Editores associados \\ Maria Alayde Mendonça e \\ Ludenulfo Lacet Cruz \\ Participantes \\ Andréa A. Brandão (RJ) \\ Angela Maria Geraldo Pierin (SP) \\ Celso Amodeo(SP) \\ Dante Marcelo Artigas Giorgi (SP) \\ Guido Rosito (RS) \\ Hilton Chaves Jr. (PE) \\ Istênio Fernandes Pascoal (DF) \\ João Cezar Mendes Moreira (SP) \\ José Luis Santello (SP) \\ José Márcio Ribeiro (MG) \\ Lilian Soares da Costa Mesquita (RJ) \\ Luis Aparecido Bortolotto (SP) \\ Marco Antonio Mota Gomes (AL) \\ Osvaldo Kohlmann Jr. (SP) \\ Paulo César Veiga Jardim(GO) \\ Raimundo Nascimento(MG) \\ Vera Koch (SP) \\ Wille Oigman (RJ)
}

Embora o conhecimento atual revele que a hipertensão arterial constitui uma síndrome caracterizada por alterações tróficas, neuroendócrinas e metabólicas, portanto muito mais que um número acima de determinado valor, o seu diagnóstico ainda é estabelecido pela correta medida da pressão arterial. Sabemos que a pressão arterial avaliada pela técnica convencional apresenta possibilidades de erros, que variam com a própria calibração do equipamento utilizado, condições inerentes ao observador, ao próprio paciente e ao ambiente em que essa verificação é realizada. $\mathrm{O}$ diagnóstico da hipertensão isolada de consultório, presente em cerca de $21 \%$ da população de hiperten sos estudada, e a recém descoberta normotensão de consultório são duas condições em que a medida da pressão arterial pela técnica convencional falha, podendo subestimar ou, mesmo, superestimar o diagnóstico da hipertensão arterial. Assim, metodologias que avaliam a pressão arterial, longe da influência do observador, podem ser úteis na avaliação diagnóstica, na avaliação da eficácia terapêutica, na pesquisa clinica, como também em outras situações especificas. Objetivando oferecer aos cardiologistas brasileiros um documento que organiza o estado atual do conhecimento sobre a utilização da MAPA (monitorização ambulatorial da pressão arterial) e da MRPA (monitorização residencial da pressão arterial), o Departamento de Diretrizes e o Departamento de Hipertensão da Sociedade Brasileira de Cardiologia, contando com a prestimosa e competente colaboração da Sociedade Brasileira de Hipertensão, do Departamento de Hipertensão da Sociedade Brasileira de Nefrologia e das diversas Regionais, organizou estas normatizacões com a simples pretensão de oferecer um suporte aos profissionais que utilizam tais metodologias em suas práticas clínicas diárias.

Graus de recomendação baseado no nível de evidência: A: Grandes ensaios clínicos aleatórios e meta-análises.

B: Estudos clínicos e observacionais bem desenhados.

C: Relatos e séries de casos.

D: Publicações baseadas em consensos e opiniões de especialistas.

\section{Normatização dos Equipamentos e Técnicas para Realização de Exames de Mapeamento Ambulatorial de Pressão Arterial (MAPA)}

1. Comportamento fisiológico da pressão arterial nas 24 horas - A pressão arterial varia devido à interação de fatores neuro-humorais, comportamentais e ambientais. $\mathrm{O}$ quadro I relaciona alguns desses principais fatores ${ }^{1}$. Observa-se uma variação contínua da pressão arterial de momento a momento, de acordo com as atividades do indivíduo, sendo que, em pacientes hipertensos, essa variabilidade da pressão arterial apresenta uma amplitude maior que nos indivíduos normais ${ }^{1}$. Durante o período de vigília, esses valores são maiores do que os obtidos durante o sono. Em função dessas variações, novos métodos surgiram para melhor avaliação do comportamento da pressão arterial, em períodos pré-determinados. Dentre eles, destacam-se: medidas 
repetidas casuais da pressão arterial, monitorização ambulatorial e/ou residencial da pressão arterial.

2.Indicações e limitações da MAPA - AMAPA permite que seja realizado um grande número de medidas - usualmente em $24 \mathrm{~h}$ - possibilitando o conhecimento do perfil de variações da pressão arterial na vigília e no sono. Entretanto, deve ser salientado que, atualmente, não existem evidências comprovadas de melhor avaliação de desfechos primários com a MAPA do que com as medidas casuais da pressão arterial. A MAPA é útil na avaliação de algumas situações clínicas, conforme o quadro II. Por outro lado, algumas condições eventualmente presentes, podem limitar a utilização da MAPA. As principais limitações ao seu uso estão no quadro III.

3. Técnicas e métodos para obtenção da pressão arterial nas $24 \mathrm{~h}$-Existem métodos diretos e indiretos de medida da pressão arterial. A MAPA se baseia na medida indireta e intermitente da pressão arterial. As medidas indiretas podem ser obtidas pelo método auscultatório ou oscilométrico.

O método auscultatório baseia-se na detecção do primeiro e do último ruído de Korotkoff, enquanto o método oscilométrico, através da identificação do ponto de oscilação máxima, determina a pressão arterial média e as pressões sistólicas e diastólicas por meio de algoritmo específi$\mathrm{co}^{2}$. O mais utilizado, atualmente, é o método oscilométrico.

4. Equipamentos -Para a utilização dos aparelhos de MAPA disponíveis no mercado, devem ser levados em

\begin{tabular}{|c|c|c|}
\hline \multicolumn{3}{|c|}{$\begin{array}{l}\text { Quadro I -Variações da pressão arterial de acordo com diversas } \\
\text { situações e atividades }\end{array}$} \\
\hline Atividades & $\begin{array}{l}\text { Pressão Sistólica } \\
\quad(\mathrm{mmHg})\end{array}$ & $\begin{array}{l}\text { Pressão Diastólica } \\
\quad(\mathrm{mmHg})\end{array}$ \\
\hline Reuniões & $+20,2$ & $+15,0$ \\
\hline Trabalho & $+16,0$ & $+13,0$ \\
\hline Caminhar & $+12,0$ & $+5,5$ \\
\hline Vestir & $+11,5$ & $+9,7$ \\
\hline Tarefas domésticas & $+10,7$ & $+6,7$ \\
\hline Telefonar & $+9,5$ & $+7,2$ \\
\hline Conversar & $+6,7$ & $+6,7$ \\
\hline Assistir a TV & $+0,3$ & $+1,1$ \\
\hline Repouso & 0 & 0 \\
\hline Dormir & $-10,0$ & $-7,6$ \\
\hline
\end{tabular}

\begin{tabular}{|l|}
\hline \multicolumn{1}{|c|}{ Quadro II - Indicações principais para o uso da mapa } \\
\hline $\begin{array}{l}\text { Hipertensão de consultório ou hipertensão do avental branco. } \\
\text { Avaliação da hipertensão arterial resistente: quando o valor da pressão ar- } \\
\text { terial permanece consistentemente elevado, apesar do uso de três ou mais } \\
\text { anti-hipertensivos comdiferentes mecanismos de ação. }\end{array}$ \\
\hline $\begin{array}{l}\text { Hipertensão arterial episódica: medidas de pressão arterial de consultório } \\
\text { normais ou elevadas, com história de medidas casuais maiores detectadas em } \\
\text { momentos episódicos, que podem ou não estar acompanhadas de sintomas. }\end{array}$ \\
\hline $\begin{array}{l}\text { Suspeita de episódios de hipotensão arterial sintomática. } \\
\text { Avaliação da eficácia terapêutica anti-hipertensiva. }\end{array}$ \\
\hline
\end{tabular}

\begin{tabular}{|l|}
\hline \multicolumn{1}{|c|}{ Quadro III - Principais limitações para o uso da mapa } \\
\hline Arritmias cardíacas. \\
Hipercinesia. \\
Braços que não permitam o perfeito ajuste do manguito. \\
Hiato auscultatório. \\
\hline
\end{tabular}

consideração, no momento da compra, os aspectos citados no quadro IV

5. Protocolo para realização do exame ${ }^{3-(\mathbb{D})}$ Pararealização da MAPA são necessárias as orientações e cuidados explicitados no quadro $\mathrm{V}$.

6. Explicações adequadas do procedimento-Opaciente que será submetido ao exame deverá receber orientações mínimas indispensáveis no sentido de obtenção de procedimento de boa qualidade. Essas orientações básicas estão listadas no quadro VI.

7. Cuidados para manutenção e calibração dos aparelhos - Deve-se exigir de cada fabricante as recomendações específicas de manutenção e calibração necessárias a cada monitor.

Esforços serão desenvolvidos pelas Sociedades Brasileiras de Cardiologia, Hipertensão e Nefrologia em busca de ações junto aos organismos oficiais, controladores de qualidade e calibração de equipamentos, como o INMETRO, para a criação de recomendações nesse sentido.

8. Reprodutibilidade do método -Para a população de indivíduos normotensos e hipertensos, independentemente do sexo ou da idade, a MAPA é um exame que apresenta boa reprodutibilidade. Os valores da pressão arterial sistólica, diastólica e média, bem como a freqüência cardíaca obtida em $24 \mathrm{~h}$, vigília e sono, apresentam resultados semelhantes em exames consecutivos realizados em curto intervalo de tempo ${ }^{4,5}(\mathrm{~B})$.

A maioria dos pacientes apresenta diminuição da pressão arterial durante o sono. A variação da pressão arterial entre os períodos de sono e vigília também apresenta boa reprodutibilidade, quando considerada como variável contínua $^{4}(\mathrm{C})$.Entretanto, a variação da pressão arterial entre os períodos de vigília e sono tem sido considerada em duas

Quadro IV - Fatores a serem considerados na aquisição de um aparelho de MAPA $^{3}$

Aparelho validado pela "British Hypertension Society" e/ou pela "US Association for the Advancement of Medical Instrumentation".

Custo do aparelho.

Custo do "software".

Informações contidas no "software".

Instruções adequadas do manual.

Custo de manutenção.

Custo do material de consumo (ex.: baterias, manguitos etc.).

Assistência técnica disponível.

Garantia. 
Quadro V - Orientação e cuidados para a realização da MAPA ${ }^{3}$

Escolher um dia representativo das atividades do paciente.

Usar manguito com largura e comprimento adequados, colocado em braço não-dominante.

Medir a pressão arterial em ambos os braços. Se a diferença da pressão arterial sistólica for menor que $10 \mathrm{mmHg}$, usar o braço não-dominante; se maior ou igual a $10 \mathrm{mmHg}$, usar o manguito no braço com maior pressão sistólica. Programar o aparelho para leituras em intervalos de 20 minutos na vigília e de 30 minutos para o período de sono, com número mínimo de 14 medidas na vigília e de 7 no sono. Por definição, o período de vigília é o compreendido entre 7 e 22 horas.

Nos aparelhos que permitem a leitura da pressão arterial, em cada momento de medida, é recomendável que tal dispositivo esteja desativado. Recomenda-se o uso de material leve e suave para separar o braço do manguito. Fazer, pelo menos, duas medidas de teste antes de liberar o paciente.

\begin{tabular}{|l|}
\hline Quadro VI - Explicações apropriadas sobre exame \\
\hline Pedir para o paciente tomar banho antes, pois não será permitido banho \\
durante o período de exame. \\
Explicar como desinsuflar manualmente o aparelho e como acionar uma me- \\
dida manual em caso de sintomas. \\
Manter o braço imóvel e relaxado ao longo do corpo durante as medidas. \\
Encorajar o indivíduo a manter suas atividades habituais durante o exame. \\
Solicitar para não deitar sobre o braço que está com o manguito instalado. \\
Anotar os horários em que o paciente dormiu e acordou; qualidade habitual \\
do sono antes e durante a realização da MAPA; horários das principais \\
atividades durante o dia, como trabalho, repouso, etc.; sintomas que surgi- \\
rem durante o exame; horários de uso de medicações, com descrição precisa \\
dos nomes e doses dos medicamentos.
\end{tabular}

categorias distintas. Quando ocorre queda da pressão arterial $\geq 10 \%$ em relação à pressão de vigília, define-se o indivíduo como com queda da pressão arterial durante o sono ou, eminglês, como "dipper". Quando a quedaé inferior a 10\%, é caracterizado como sem queda da pressão arterial durante o sono ou, em inglês, como "non-dipper". A reprodutibilidade do caráter "dipper" "e "non-dipper" tem sido questionada na literatura devido à chance de $30 \%$ a $50 \%$ dos indivíduos mudarem de categoria em exames subseqüentes ${ }^{6}(\mathrm{C})$. Essa aparente controvérsia se deve às limitações advindas da utilização de pontos de cortes arbitrários, nesta condição $10 \%$, para representar variáveis contínuas. Assim sendo, o fenômeno da variação da pressão arterial entre a vigília e o sono deve ser considerado em seu valor absoluto (em $\mathrm{mmHg}$ ) e percentual.

9. Valores de normalidade para as pressões obtidas com a MAPA - À semelhança da medida casual da pressão arterial, os critérios de normalidade dos valores de pressão na MAPA são arbitrários. O quadro VII expressa os valores considerados normais, intermediários e anormais para a MAPA. Para a população adulta, as medidas normais e anormais disponíveis e recomendadas representam apenas um guia para a interpretação do exame. Níveis mais baixos de pressão podem ter relevância clínica em pacientes com múltiplos fatores de risco ${ }^{7}(\mathrm{D})$. É importante salientar que não existem estudos longitudinais com o uso da MAPA na população brasileira.

\begin{tabular}{|llll|}
\hline \multicolumn{4}{|c|}{ Quadro VII - Valores de normalidade para MAPA } \\
\hline Média da pressão & Normal & Intermediário & Anormal \\
\hline Sistólica & & & \\
Vigília & $<135$ & $135-140$ & $>140$ \\
Sono & $<120$ & $120-125$ & $>125$ \\
24 horas & $<130$ & $130-135$ & $>135$ \\
\hline Diastólica & & & \\
Vigília & $<85$ & $85-90$ & $>90$ \\
Sono & $<75$ & $75-80$ & $>80$ \\
24 horas & $<80$ & $80-85$ & $>85$ \\
\hline
\end{tabular}

As médias sistólicas e diastólicas na vigília apresentam, geralmente, valores mais elevados, quando comparados aos obtidos por medida casual, enquanto as médias pressóricas sistólicas e diastólicas durante o sono mostram valores inferiores àqueles obtidos casualmente.

10. Crianças - Dados de MAPA em crianças têm demonstrado boa correlação com idade cronológica, altura, peso e frequiência cardíaca, além de boa reprodutibilidade ${ }^{8}(\mathrm{C})$.

Belsha e cols ${ }^{9}$ (C) compararam, através da MAPA, o perfil de pressão arterial de vigília e sono de crianças e adolescentes normotensos e hipertensos, demonstrando que os pacientes hipertensos apresentam médias pressóricas mais elevadas, com perfil pressórico de $24 \mathrm{~h}$ semelhante aos seus pares normotensos. Não existem critérios estabelecidos de normalidade para as variáveis obtidas. Para cálculo das cargas pressóricas de vigília, utiliza-se, como limite superior de normalidade, o percentil 95 para sexo e idade da curva de referência da pressão arterial casual, sendo usados valores $10 \%$ menores para as pressões de sono.

Contudo, a avaliação pela MAPA de crianças e adolescentes com doenças de base com risco associado de desenvolvimento de hipertensão arterial tem sido descrita como promissora em pacientes com diabetes mellitus tipo $1^{10}(\mathrm{C})$, doença policística autossômica dominante ${ }^{11}(\mathrm{C})$, na insuficiência renal crônica dialítica ${ }^{12,13}(\mathrm{C})$ e em pacientes transplantados renais $^{14}$ (C). Nessas situações clínicas, a MAPA tem se mostrado mais útil para o diagnóstico e manejo da hipertensão arterial do que a pressão arterial de consultório, por fornecer dados sobre alterações do perfil pressórico durante o sono, reclassificar pacientes quanto ao diagnóstico de hipertensão arterial obtido pela medida em consultório e apresentar maior correlação com o desenvolvimento de lesões de órgãos-alvo.

11. Grávidas - A maioria dos estudos de parâmetros circadianos da pressão arterial durante a gravidez demonstra a preservação do padrão vigília-sono e variabilidade global semelhante àquela observada em mulheres não-grá$\operatorname{vidas}^{15,16}(\mathrm{C})$. Igualmente, observa-se diminuição linear da pressão arterial sistólica, diastólica e média até a $22^{2}$ semana de gestação, seguida por aumento progressivo até o dia do $\operatorname{parto}^{17-19}(\mathrm{C})$.

Variações da pressão arterial na pré-eclâmpsia têm sido reconhecidas há muito tempo. Entretanto, a atenuação 
ou inversão do padrão vigília-sono, inicialmente descrita, apresenta baixa sensibilidade. Isto é, muitos casos de préeclâmpsia, definidos sob critério clínico ${ }^{20}(\mathrm{C})$ ou histológico $\operatorname{renal}^{21}(\mathrm{C})$, não apresentam as alterações mencionadas relativas ao ritmo circadiano.

A possibilidade de antecipar o desenvolvimento de pré-eclâmpsia representaria o maior benefício que a MAPA poderia trazer à propedêutica na mulher gestante. Níveis de pressão arterial média $>95 \mathrm{mmHg}$ e de pressão arterial diastólica superiores a $80 \mathrm{mmHg}$ no $1^{\circ}$ trimestre e $>90 \mathrm{mmHg}$ e 75 $\mathrm{mmHg}$ respectivamente no $2^{\circ}$ trimestre, embora dentro da faixa normal, foram inicialmente identificados como preditivos do desenvolvimento de pré-eclâmpsia ${ }^{22}(\mathrm{C})$, porém esses dados não foram convincentemente confirmados, em boa medida devido à ampla sobreposição de valores de pressão entre as pacientes que efetivamente desenvolvem pré-eclâmpsia e as que não desenvolvem ${ }^{23}(\mathrm{C})$.

12. Fenômeno do avental branco - Os níveis da pressão arterial medidos em consultório podem ser maiores, semelhantes ou menores do que os obtidos em vigília pela MAPA. Essas diferenças eventuais possibilitam a classificação dos pacientes em duas diferentes categorias: hipertensão do avental branco ${ }^{24}$ (B) ou normotensão do avental branco $^{25}$ (B) (fig. 1).

Registra-se, igualmente, o efeito do avental branco ${ }^{24}$ (B). Conforme definições e valores expressos no quadro VIII, define-se cada situação específica.

Há evidências de que a hipertensão do avental branco possa não ser tão benigna como se pensava, ocorrendo maior possibilidade de acometimento de órgãos-alvo que em populações normotensas, porém em menor escala do que em hipertensos ${ }^{26}$ (B). Por outro lado, alguns autores demonstram semelhante possibilidade de eventos cardio-

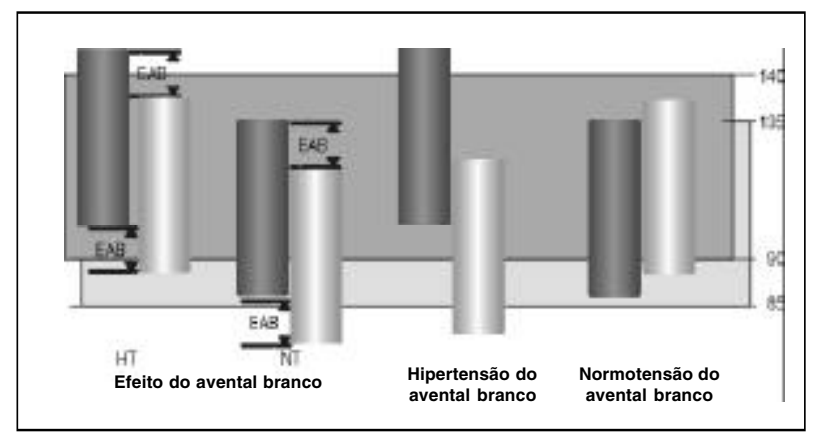

Figura 1 - Classificação vasculares para esse grupo de indivíduos, quando comparados com uma população de normotensos ${ }^{27}(\mathrm{~B})$.

Não há características clínicas específicas que auxiliem neste diagnóstico, embora sua prevalência aumente com a idade, notadamente no sexo feminino. Não existem evidências de benefícios de intervenções medicamentosas neste grupo de pacientes; entretanto, o paciente deve ser considerado no contexto de seu risco cardiovascular global, permanecendo em seguimento clínico.

Significado clínico e prognóstico da normotensão do avental branco -Recentemente, destacou-se um grupo de pacientes cuja pressão arterial é elevada pela MAPA, mas apresenta valores normais na medida casual.

Estudos restritos têm sugerido que esses pacientes têm maior prevalência de lesões de órgãos-alvo do que indivíduos normotensos nos dois métodos de medida da pressão $\operatorname{arterial}^{25,29}(\mathrm{C})$.

13. Papel da MAPA na avaliação prognóstica em pacientes hipertensos -Por serem mais reprodutíveis e melhor estimar a variável pressão arterial, os valores de pressão arterial obtidos pela MAPA se correlacionam mais fortemente com lesões de órgãos-alvo e morbidade e mortalidade do que as medidas casuais da pressão arterial.

Os valores médios de pressão arterial sistólica e diastólica de $24 \mathrm{~h}$, de vigília e de sono, apresentam correlação positiva com índice de massa de ventrículo esquerdo, lesões isquêmicas encefálicas e microalbuminúria. Em idosos, avaliados no estudo "Syst-Eur", a variável que apresentou melhor correlação com eventos cardiovasculares maiores, como acidente vascular encefálico, infarto agudo do miocárdio e óbito, foi a pressão arterial sistólica do sono, seguida da pressão arterial sistólica de 24 h e da pressão arterial sistólica de vigília. O estudo de Ohasama ${ }^{28}$ (B) encontrou achados semelhantes.

A intensidade da queda da pressão arterial no sono mostra correlação inversa com a massa de ventrículo esquerdo e microalbuminúria. Em relação a lesões isquêmicas encefálicas, os indivíduos com atenuação da queda no sono $(<10 \%)$ ou com queda noturna exagerada $(>20 \%)$ apresentam maior número de áreas isquêmicas à ressonância nuclear magnética cerebral.

A variabilidade da pressão arterial estimada pelo desvio padrão (coeficiente de variação percentual) de medidas contínuas da pressão arterial batimento a batimento apresenta correlação positiva com lesões de órgãos-alvo. Entre-

\begin{tabular}{|c|c|c|c|c|}
\hline \multicolumn{5}{|c|}{ Quadro VIII - Conceitos, classificações e prevalências da hipertensão, normotensão e efeito do avental branco } \\
\hline Classe & Conceito & $\begin{array}{c}\text { Pressão } \\
\text { de consultório }\end{array}$ & $\begin{array}{c}\text { Pressão } \\
\text { de vigília }\end{array}$ & $\begin{array}{c}\text { Prevalência } \\
(\%)\end{array}$ \\
\hline Hipertensão do avental branco & Pressão consultório > pressão vigília & $>140 / 90$ & $<135 / 85$ & $20-30$ \\
\hline Normotensão do avental branco & Pressão consultório < pressão vigília & $<140 / 90$ & $>140 / 90$ & $10-20$ \\
\hline \multirow[t]{2}{*}{ Efeito do avental branco } & Pressão consultório > pressão vigília & Normotenso & Normotenso & \\
\hline & $(>20 / 10 \mathrm{mmHg})$ & Hipertenso & Hipertenso & \\
\hline
\end{tabular}


tanto, por possível limitação da MAPA, essa associação não foi demonstrada com o desvio padrão (coeficiente de variação percentual) obtido pela MAPA de 24h.

\section{Avaliação dos dados obtidos com a MAPA - Para a} análise dos dados obtidos com a MAPA ${ }^{29}$, há necessidade inicial de se avaliar a qualidade do exame. Os dados obtidos e avaliáveis são: médias pressóricas; diferenças de pressão vigília-sono; correlações entre pressões e atividades, sintomas e medicamentos; presença de picos tensionais e episódios de hipotensão. Outros parâmetros são obtidos, mas, ou não existem critérios definitivos para a sua interpretação, como pressão arterial média, pressão de pulso e variabilidade, ou são mal estimados pelos equipamentos disponíveis, tais como frequiência cardíaca e, finalmente, aqueles que têmimportância clínica limitada, como, por exemplo, a carga pressórica (quadro IX).

15. Validade do procedimento - Thijs e $\operatorname{col}^{30}(B)$ analisaram a validade do exame baseado no número mínimo de medidas necessárias em $24 \mathrm{~h}$ e concluíram que esse número mínimo deveria ser de, pelo menos, uma medida a cada $30 \mathrm{~min}$. Considerando-se uma perda média de $20 \%$, recomenda-se a realização das medidas, no mínimo, a cada $20 \mathrm{~min}$ durante a vigília e a cada $30 \mathrm{~min}$ no período de sono. A exigência mínima é de, pelo menos, 14 medidas na vigíliae $7 \mathrm{du}$ rante o sono. Em determinadas situações, como perda de medidas em horários não-relevantes, a juízo clínico, um número de medidas abaixo do preconizado pode ser aceitável.

16. Médias de pressão arterial-Dentre os parâmetros obtidos pela MAPA, as médias de pressão arterial são os melhores dados a serem analisados, por apresentarem maiores índices de correlação com diagnóstico, lesão em órgãos-alvo e prognóstico cardiovascular, tendo sido o único parâmetro relacionado à mortalidade, de acordo com o estudo de Ohkubo e $\operatorname{cols}^{28}$ (B). A análise dos períodos de 24h, vigília e sono é considerada essencial para avaliação das médias de pressão. Períodos específicos, como as primeiras

\begin{tabular}{|l|}
\hline \multicolumn{1}{|c|}{ Quadro IX - Avaliação dos dados obtidos com a MAPA } \\
\hline $\begin{array}{l}\text { Dados avaliáveis } \\
\text { médias pressóricas } \\
\text { diferenças vigília / sono } \\
\text { relações pressão-atividade / sintomas / medicamentos } \\
\text { picos tensionais } \\
\text { hipotensão }\end{array}$ \\
\hline $\begin{array}{l}\text { Sem critérios definidos para interpretação } \\
\text { pressão arterial média } \\
\text { pressão de pulso } \\
\text { variabilidade }\end{array}$ \\
\hline $\begin{array}{l}\text { Mal estimado pelos equipamentos } \\
\text { frequêencia cardíaca }\end{array}$ \\
\hline $\begin{array}{l}\text { Importância clínica limitada } \\
\text { cargas pressóricas }\end{array}$ \\
\hline
\end{tabular}

horas após o despertar ou na sesta, não têm ainda sua importância clínica bem estabelecida. Recomenda-se que esse parâmetro seja interpretado de acordo com os valores expressos no quadro VII, estacando-se a normalidade ou não dos valores obtidos. Deve-se alertar para a presença de médias consideradas intermediárias. Tanto as médias sistólicas quanto diastólicas, isoladas ou simultaneamente, têm valor clínico.

17. Diferenças de pressão vigília-sono-É fundamental para a definição dos períodos de vigília e de sono a anotação precisa dos horários em que o indivíduo submetido ao exame dormiu e acordou. Esses dados devem estar claramente anotados no diário de atividades.

A referência, pelo paciente, de sono intensamente perturbado na vigência do exame deve ser considerada no momento da interpretação das variações das pressões vigília-sono.

Usualmente, encontra-se queda da pressão sistólica e diastólica durante o sono quando comparado ao período de vigília. Foi observado em hipertensos que queda inferior a $10 \%$ relacionava-se a pior prognóstico cardiovascular ${ }^{31}(\mathrm{~B})$. Em indivíduos normotensos, a ausência de queda da pressão arterial durante o sono não tem significado clínico confirmado. Shimada e $\operatorname{cols}^{32}$ (B) demonstraram, em idosos hipertensos com descenso superior a $20 \%$, que houve aumento de risco cardiovascular, especialmente para a ocorrência de acidente vascular encefálico.

Vale lembrar que a inversão do comportamento fisiológico da pressão vigília-sono ou ausência de queda podem estar relacionadas a determinadas situações, tais como distúrbio do sono provocado pelo exame, controle inadequado da pressão em pacientes tratados, em algumas situações, de hipertensão secundária, apnéia do sono, disautonomia e uso de algumas drogas, como a ciclosporina.

18. Correlações de pressões com atividades, sintomas e medicamentos - Para a devida análise desses itens, é essencial o preenchimento detalhado do diário de atividades, sendo de grande importância a anotação dos horários em que o paciente dormiu e acordou, usou medicamentos ou bebidas alcoólicas, café e tabaco.

A presença de sintomas, horário de trabalho ou atividade física também precisam ser detalhadamente descritos ${ }^{33}(D)$.

\section{Picos hipertensivos e episódios de hipotensão -} Elevações significativas da pressão arterial de forma progressiva, em, pelo menos, duas medidas, atingindo um pico claramente acima das variações anterior e posterior, configuram pico hipertensivo. Vale a pena ressaltar que valores elevados e isolados, na maioria das vezes, representam artefatos, portanto, sem significado.

Episódios sintomáticos de diminuição da pressão arterial podem ser importantes, especialmente nas seguintes situações: ação medicamentosa, síncope, lipotímia pósprandial em idosos, hipotensão postural, disautonomia e diabetes mellitus, entre outros. Medidas isoladas e assin- 
tomáticas de diminuição acentuada da PA também podem ser decorrentes de artefatos técnicos.

20. Pressão arterial média, pressão de pulso e variabilidade - A pressão arterial média é obtida pelo exame, com aplicabilidade restrita à pesquisa clínica.

A despeito da grande importância clínica que se tem dado à pressão de pulso, com base nas medidas casuais, com fortes evidências de implicações prognósticas, não há até o momento critérios para a interpretação desse parâmetro pela MAPA.

Sabe-se que a variabilidade da pressão arterial tem grande correlação prognóstica com eventos cardiovasculares e desenvolvimento de lesão em órgãos-alvo. Entretanto, a avaliação apropriada da variabilidade é somente possível através de registro contínuo da pressão arterial (batimento a batimento), o que não é obtido pelo método usual de registro da pressão arterial em $24 \mathrm{~h}$. O desvio padrão das médias de pressão arterial obtido pela MAPA vem sendo inadequadamente utilizado como indicativo da variabilidade da pressão arterial. Entretanto, até o momento não há critérios de normalidade para sua interpretação.

21. Frequiência cardíaca - Apesar de registrarem a freqüência cardíaca, os equipamentos utilizados para a MAPA não são apropriados para a obtenção desse parâmetro, não devendo, portanto, ser considerado, exceto nos equipamentos capazes de registrar simultaneamente o eletrocardiograma em $24 \mathrm{~h}$.

22. Cargas pressóricas - Embora tenha definição proposta desde $1988^{34}(\mathrm{C})$ - percentagens de medidas acima dos valores de referência-, este critério suporta várias críticas.

Dentre elas, uma das mais consistentes está relacionada ao fato de que o mesmo valor de cargas pressóricas pode significar diferentes comportamentos estimados pelas médias de pressão ${ }^{35}$.

Mesmo havendo documentação sobre a relação direta entre valores de cargas, especialmente acima de $50 \%$, e lesões em órgãos-alvo, há a tendência, nas mais recentes diretrizes para utilização da MAPA, de não se considerarem na interpretação clínica os valores de cargas pressóricas ${ }^{36-38}(\mathrm{D})$.

Assim, pode-se julgar igualmente inapropriada a consideração dessa variável na interpretação rotineira do exame.

23. Produção de relatórios - O relatório da MAPA deve conter obrigatoriamente os itens apontados na no quadro $X^{39}(D)$.

Não se deve estabelecer, em conformidade com as informações hoje disponíveis, diagnóstico de hipertensão utilizando-se a MAPA, pois este é um diagnóstico clínico. Quando indicada para fins de avaliação do comportamento da pressão em $24 \mathrm{~h}$, deve-se apenas considerar nas conclusões: comportamento normal, intermediário ou anormal da pressão em 24h. Para fins de avaliação da eficácia terapêutica, deve-se concluir que: as medicações referidas como utilizadas estejam ou não exercendo adequado controle da pressão arterial nas $24 \mathrm{~h}$.
Quadro X - Itens que necessariamente deverão ser avaliados em um relatório de MAPA

Qualidade do exame

Médias de pressão

Diferenças de pressão vigília-sono

Correlações entre pressões e atividades, sintomas e medicamentos

Picos tensionais e episódios de hipotensão

Comentários

Conclusões

\section{Normatização dos Equipamentos e Técnicas para Realização de Exames de Monitorização Residencial da Pressão Arterial(MRPA)}

1. Importância da monitorização residencial da pressão arterial (MRPA) - A medida da pressão arterial no consultório, apesar de considerada procedimento padrão para o diagnóstico de hipertensão e para o seguimento de pacientes hipertensos, está sujeita a inúmeros fatores de erro, sendo o mais importante dentre eles a influência do observador. Além disso, tal medida propicia um número reduzido de leituras, que não apresenta boa reprodutibilidade ao longo do tempo.

A medida residencial realizada durante vários dias pelo paciente ou familiar devidamente treinado constitui uma alternativa útil para evitar esses inconvenientes. Essa não é uma técnica inovadora, pois, já em $1940^{40}$, havia sido demonstrado que a medida residencial apresentava valores de 30 a $40 \mathrm{mmHg}$ mais baixos do que a medida no consultório. Comparando-se os valores de medida da pressão no consultório com a MAPA e a medida residencial, verificou-se que a residencial apresentou valores mais baixos do que a MAPA e a pressão de consultório ${ }^{41}$.

Com o desenvolvimento de equipamentos compactos, confiáveis, validados e de preços mais acessíveis, o procedimento se tornou viável para uso em larga escala na prática clínica diária e na pesquisa.

2. Indicações, vantagens e limitações da MRPA-Dentre as vantagens da MRPA em relação às medidas casuais (como a de consultório) está a melhoria dos índices de adesão ao tratamento, o que levou vários organismos internacionais a sugerirem o uso rotineiro de tal procedimento.

3. Equipamentos- A medida da pressão arterial pelo paciente ou familiar pode ser realizada através de: esfigmomanômetro de coluna de mercúrio; esfigmomanômetro aneróide; aparelhos oscilométricos semi autométicos com deflagração manual de medida no braço, no pulso ou no dedo ${ }^{53}$.

O esfigmomanômetro de coluna de mercúrio não é apropriado para medida pelo paciente ou familiar, devido às dificuldades do método indireto de medida com técnica auscultatória, além de o mercúrio estar sendo gradativamente banido do uso médico devido a sua toxicidade ${ }^{54}$. $\mathrm{O}$ aneróide apresenta as mesmas dificuldades do método indireto com técnica auscultatória, além da descalibração mais freqüente do que o de coluna de mercúrio. 
Com relação aos aparelhos oscilométricos semi ou automáticos com deflagração manual, os de medida no braço são os mais recomendados e confiáveis (quadro XI). Os que medem a pressão no pulso apresentam limitações devido à necessidade de colocação do pulso ao nível do coração, além de erros devido à flexão ou hiperextensão do pulso durante a medida. Aqueles que medem no dedo, apesar de convenientes para o paciente, não são recomendáveis devido à baixa confiabilidade ${ }^{55}$. É fundamental que os aparelhos empregados sejam validados de acordo com as normas da "British Hypertension Society" (BHS) e "Association for Advancement of Medical Instruments" (AAMI) ou outros critérios aceitos internacionalmente ${ }^{56}$. Além disso, é importante que o equipamento tenha sua calibração testada contra aparelho de coluna de mercúrio, pelo menos, anualmente.

Outro aspecto relacionado ao equipamento diz respeito à necessidade do uso de manguito de tamanho adequado ao braço do paciente.

4. Protocolos, procedimentos e instruções ao paciente - Vários protocolos de MRPA têm sido utilizados ${ }^{53-57}$. Recomendam-se, pelo menos, duas medidas pela manhã, antes da tomada das medicações e do desjejum, e duas à noite, antes do jantar ou três horas depois, para evitar a redução pós-prandial da pressão arterial, durante, no mínimo, três dias consecutivos de atividades habituais, desprezando-se as medidas do primeiro dia. As instruções ao paciente encontram-se nos quadros XIII e XIV.

5. Valor clínico da MRPA - As indicações do emprego do MRPA acham-se nos quadros XIV e XV

Quadro XI - Vantagens da MRPA realizada com aparelho semi automático em comparação às medidas de consultório

Oferece maior número de medidas ${ }^{42}$

Apresenta boa aceitabilidade inclusive por idosos e muito idosos ${ }^{43}$

Favorece maior adesão ao tratamento ${ }^{44}$

Mostra boa reprodutibilidade ${ }^{45}$

Avalia a pressão sem influência do observador e do ambiente do consultório ${ }^{46}$

Atenua os erros e preferências do observador ${ }^{47}$

Apresenta menor efeito placebo ${ }^{48}$

Mostra melhor correlação com lesão de órgãos-alvo ${ }^{46}$

Possibilita armazenamento, impressão e transmissão a distância das leituras

obtidas $^{44}$

Diminui o número de visitas ${ }^{49}$

Os aparelhos têm menor custo ${ }^{50}$

Quadro XIII - Instruções ao paciente para a realização da MRPA

Efetuar a medida na posição sentada após 1-2 minutos de repouso

Estar em ambiente tranqüilo com temperatura agradável

Utilizar preferencialmente o braço esquerdo apoiado na altura do coração com a palma da mão voltada para cima

Anotar os valores realmente obtidos quando empregar equipamentos que não armazenam ou imprimem os dados

Preencher o diário com as principais atividades, tais como horário de refeições, tomada de medicamentos ou sintomas

Realizar medidas adicionais quando surgirem sintomas ou situações especiais

Seguir as instruções do fabricante do aparelho quanto à deflagração do procedimento

\section{Quadro XIV - Indicações da MRPA realizada com aparelho semi ou automático $^{47,51,52}$}

Seguimento do hipertenso do avental branco Quantificação do efeito do avental branco

Avaliação da eficácia terapêutica anti-hipertensiva

Utilização em ensaios clínicos

\subsection{Diagnóstico da hipertensão do avental branco -}

A medida residencial da pressão arterial, por ser isenta do efeito do observador, possibilita o diagnóstico do efeito e da hipertensão do avental branco, mais freqüentemente observados nas hipertensões leves. No entanto, Stergiou e $\mathrm{cols}^{58}$ mostraram que a medida residencial não foi apropriada como alternativa à MAPA no diagnóstico da hipertensão do avental branco, podendo, porém, ser útil como método de rastreamento desse fenômeno e de acompanhamento em longo prazo dos hipertensos nessas condições, devido a sua alta especificidade e baixo custo.

\subsection{Avaliação da eficácia da terapêutica anti-hiper-} tensiva - Graças ao baixo custo ${ }^{50}$, boa aceitabilidade ${ }^{59}$, facilidade de manuseio, possibilidade de avaliação em longo prazo e monitorização a distância ${ }^{60}$, a medida residencial pode ser bastante apropriada para avaliação da eficácia da terapêutica anti-hipertensiva. Além disso, pode ser utilizada na avaliação do efeito de drogas anti-hipertensivas em ensaios clínicos, diminuindo o número de pacientes necessário ao estudo ${ }^{61}$.

A medida residencial é particularmente útil na avaliação da eficácia da terapêutica em pacientes hipertensos resistentes às medidas de consultório, por poder observar o efeito do avental branco ${ }^{62}$.

5.3. Prognóstico do hipertenso - Dois estudos prospectivos importantes avaliaram o papel prognóstico da medida residencial. Assim, o estudo de Ohasama ${ }^{62}$ analisou grande número de indivíduos durante vários anos, tendo observado mortes como desfecho, e demonstrou que a medida residencial apresenta correlação com mortalidade cardiovascular total, morbidade por acidente vascular encefálico e mortalidade não-cardiovascular. Ao comparar a medida residencial com a casual de consultório, os autores verificaram que aquela mostrou poder preditivo mais forte do que esta.

No estudo "Tecumseh" ${ }^{3}$ foi sugerido que a medida re-

Quadro XV - Limitações da MRPA realizada com aparelho semi ou automático $^{47,51,52}$

Impossibilidade de medir a pressão durante o sono

Tempo despendido na instrução do paciente e/ou familiares

Número reduzido de estudos de normalidade e prognóstico

Grande número de equipamentos não-validados

Pacientes arrítmicos, obesos e crianças

Possibilidade de o paciente auto-ajustar a medicação, induzido pelo valor da leitura 
sidencial pode ter valor preditivo para o desenvolvimento de hipertensão e disfunção diastólica do ventrículo esquerdo.

Vários estudos têm mostrado melhor correlação da medida residencial com lesões de órgãos-alvo. Em nosso meio, foi demonstrada melhor correlação da medida residencial com índice de massa de ventrículo esquerdo quando comparada com a medida de consultório ${ }^{41}$.

6. Critérios de normalidade - Análise de banco de dados internacional, considerando medidas de manhã e noite, mostrou valores de normalidade $\leq 135 / 85 \mathrm{mmHg}$ e anormais acima de 140/90 $\mathrm{mmHg}$, havendo zona de incerteza entre esses limites ${ }^{64}$.

Outro critério sugerido é empregar valores de medida residencial correspondentes a valores de medida de consultório. Assim, o valor correspondente a 130/85 mmHg no consultório seria $125 / 80 \mathrm{mmHg}$, e 140/90 mmHg no consultório corresponderia a $133 / 86 \mathrm{mmHg}$ na $\mathrm{MRPA}^{65}$.

O valor limite de 135/85 mmHg tem sido recomendado por organismos internacionais, como o JNC-VI, e nacionais, como o III Consenso Brasileiro de Hipertensão Arterial ${ }^{66}$. Em situações especiais, como no diabetes mellitus, em crianças e gestantes ainda não estão estabelecidos os critérios de normalidade. tórios

7. Interpretação dos dados obtidos e produção de rela-

A) Descrição do protocolo utilizado - Como o protocolo depende do objetivo do exame, a sua descrição é fundamental.
B) Qualidade do procedimento - O registro deverá ser aceito para interpretação quando atingir, pelo menos, $80 \%$ de leituras válidas em relação às leituras previstas.

Deverão ser excluídas as medidas aberrantes, tais como diastólica $>140 \mathrm{mmHg}$ e $<40 \mathrm{mmHg}$, sistólica $<$ de 70 $\mathrm{mmHg}$ e $>250 \mathrm{mmHg}$ e pressão de pulso $<20 \mathrm{mmHg}$.

C) Médias de pressão - De acordo com o banco de dados internacional, as médias de pressão são consideradas normais quando $\leq 135 / 85 \mathrm{mmHg}$. Recomendamos considerar exame anormal quando as médias estiverem > 135/85 mmHg para registros de, no mínimo, três dias, desprezandose o primeiro dia.

No relatório deve-se citar, além das médias diárias e total do registro, a ocorrência de medidas mais elevadas no primeiro dia de registro, que podem estar relacionadas à reação de alarme, bem como medidas de outros dias que contrastem com a média de todas as medidas. Também é interessante analisar a média dos valores de pressão dos períodos da manhã e noite, principalmente em pacientes sob terapêutica medicamentosa.

D) Correlação com sintomas e medicação -Deve ser descrita no relatório, de acordo com as anotações do diário do paciente. Assim, a medida residencial surge como mais uma ferramenta que pode auxiliar no manejo do paciente hipertenso, facilitando não só a identificação da real elevação dos níveis tensionais, como também melhor avaliando a efetividade do tratamento instituído e favorecendo uma melhor adesão ao tratamento.

\section{Referências}

1. Clark LA, Denby L, Pregibon D, Harshfield GA, Pickering TG, Blank S, Laragh JH. Effects of activity on diurnal variations of blood pressure. J Chron Dis, 1987; 40: 671-81.

2. Mauck GB, SmithCR, Geddes LR etal. The meaning of the point of maximum oscillations in cuff pressure in the indirect measurement of blood pressure II. J Biomech Eng, 1980;102:28-33.

3. O'Brien E, Coats A, Owens P, Petrie J, Padfield PL, Littler WA, Swiet M, Mee F. Use and interpretation of ambulatory blood pressure monitoring: recommendations of the British Hypertension Society. BMJ, 2000;320:1128-34

4. Chaves Jr HC. Monitorização Ambulatorial da Pressão Arterial (MAPA-24h): sua reprodutibilidade e importância na construção de modelos para estimar a hipertrofia cardíaca. São Paulo; 2000. (Tese de doutorado - Faculdade de Medicina da Universidade de São Paulo).

5. Omboni S, Parati G, Palatini P, Vanasia A, Muiesan ML, Cuspidi C, Mancia G. Reproducibility and clinical value of nocturnal hypotension: prospective evidence from the SAMPLE study. J Hypertens, 1998; 16: 733-88.

6. Mochizuki Y, Okutani M, Donfeng Y, Ywasaki H, Takusagawa M, Kohno I, Mochizuki S, Umetani K, Ishii H, Ijiri H, Komori S, Tamura K. Limited reproducibility of circadian variation in blood pressure dippers and non-dippers. Am J Hypertens, 1998; 11: 403-9

7. Ramsay LE, Williams B, Johnston GD, MacGregor GA, Poston L, Potter JF. Guidelines for management of hypertension: report of the third working party of the British Hypertension Society. J Hum Hypertens, 1999; 13: 569-92

8. Lurbe E, Aguilar F, Gomez A, Tacons J, Alvarez V, Redon J. Reproducibility of ambulatory blood pressure monitoring in children. J Hypertens, 1993; 11:S288-

9. Belsha CW, Spencer III HJ, Berry Pl, Plummer JK, Wells TG. Diurnal blood pressure patterns in normotensive and hypertensive children and adolescents. J Hum Hypert,1997; 11: 801-6.
10. Sochett EB, Poon I, Balfe W, Daneman D. Ambulatory blood pressure monitoring in insulin dependent diabetes mellitus adolescents with and without microalbuminuria. J Diab Complications, 1998; 12: 18-23

11. Seeman T, Sikut M, Konrad M, Vondrichova H, Janda J, Scharer K. Blood pressure and renal function in autosomal dominant polycystic kidney disease. Pediatr Nephrol, 1997; 11: 592-6.

12. Lingens N, Soergel M, Chantal L, Busch C, Lemmer B, Schörer K. Ambulatory blood pressure monitoring in paediatric patients treated by regular haemodialysis and peritoneal dialysis. Pediatr Nephrol, 1995; 9: 167-72.

13. Koch VH, Furusawa E, Ignez E, Okay Y, Mion Jr D. Ambulatory blood pressure monitoring in chronically dialysed pediatric patients. Blood Pressure Monit, 1999; 4: 213-6.

14. Calzolari A, Giordano U, Matteucci MC, Pastore E, Turchetta A, Rizzoni G, Alpert B. Hypertension in young patients after renal transplantation: ambulatory blood pressure monitoring versus casual blood pressure. Am J Hypert, 1998; 11: 497-501.

15. Contard S, Chanudet X, Coisne D, Battistella P, Marichal JF, Pitiot M, de Gaudemaris R, Ribstein J. Ambulatory monitoring of blood pressure in normal pregnancy. Am J Hypertens, 1993; 6: 880-4.

16. Brown MA, Robinson A, Bowyer L, Buddle ML, Martin A, Hargood JL, Cario GM. Ambulatory blood pressure monitoring in pregnancy: what is normal? Am J Obstet Gynecol, 1998; 178: 836-42

17. Murnaghan GA, Mitchell RH, Ruff S. Circadian variation of blood pressure in pregnancy. In: Bonnar J, MacGillivray I, Symonds EM (eds.). Pregnancy hypertension. Lancaster: MTP Press, 1980; 107-12.

18. Clark S, Hofmeyr GJ, Coats A. Ambulatory blood pressure monitoring during pregnancy: validation of the TM 2420 monitor. Obstet Gynecol, 1991;77: 152-5.

19. Halligan A, O'Brien E, O'Malley K. Twenty four hour ambulatory blood pressure measurement in a primigravid population. J Hypert, 1993; 11: 869:73 
20. Peek M, Shennan A, Halligan A, Lambert PC, Taylor DJ, De Swiet M. Hypertension in pregnancy: which method of blood pressure measurement is most predictive of outcome? Obstet Gynecol, 1996; 88: 1030-3

21. Hermida RC, Ayala DE. Diagnosing gestational hypertension and preeclampsia with the 24-hour mean of blood pressure. Hypertension, 1997; 30: 1531-7.

22. Olofsson P, Persson K. A comparison between conventional and 24 - hour automatic blood pressure monitoring in hypertensive pregnancy. Acta Obstet Gynecol Scand, 1995; 74: 429-33

23. Luders C, Mion Jr D, Kahhale S, Zugaib M, Sabbaga E, Marcondes M. Nocturnal blood pressure fall in pre-eclamptic patients, confirmed by renal biopsy. Am J Hypertens, 1994; $7: 113$.

24. Pickering TG, James GD, Boddie C, Harshfield GA, Blank S, Laragh JH. How common is white coat hypertension? JAMA, 1988; 259: 225-8.

25. Liu JE, Roman MJ, Pini R, Schwartz JE, Pickering TG, Devereux RB. Cardiac and arterial target organ damage in adults with elevated ambulatory and normal office blood pressure. Ann Intern Med, 1999; 131: 564-72.

26. Julius S, Mejia A, Jones K, Krause L, Schork N, van de Vem C. "White coat" versus "sustained" borderline hypertension in Tecumseh, Michigan. Hypertension, 1990; 16: 617: 23.

27. Cavallini MC, Roman MJ, Pickering TG, Schwartz JE, Pini R, Devereux RB. Is white coat hypertension associated with arterial disease or left ventricular hypertrophy? Hypertension, 1995; 26: 413-9.

28. Ohkubo T, Hozawa A, Nagai K, Kikuya M, Tsuji I, Ito S, Satoh H, Hisamichi S, Imai Y. Prediction of stroke by ambulatory blood pressure monitoring versus screening blood pressure measurements in a general population: the Ohasama study. J Hypertens, 2000; 18: 847-54

29. Schillaci G, Verdecchia P, Sacchi N, Bruni B, Benemio G, Pede S, Porcellati C. Clinical relevance of office underestimation of usual blood pressure in treated hypertension. Am J Hypertens, 2000; 13: 523-8.

30. Thijs L, Staessen J, Fagard R, Zacariah P, Amery A. Number of measurements required for the analysis of diurnal blood pressure profile. J Human Hypertens, 1994; 89: 239-44.

31. O' Brien, Sheridan J, O' Malley K. Dippers and non-dippers. Lancet, 1988; 13 397.

32. Kario K, Matsuo T, Kobayashi H, Imiya M, Matsuo M, Shimada K. Nocturnal fall of blood pressure and silent cerebrovascular damage in elderly hypertensive patients. Advanced silent damage in extrure dippers. Hypertension, 1996; 27 : $130-5$.

33. Mion Jr D, Nobre F. Manual de instalação e cuidados. Lemos Editorial, 1998

34. Zacariah PK, Sheps SG, Ilstrip DM et al. Blood Pressure: a better determinant of hypertension. Mayo Clin Proc, 1988; 63: 1085-91.

35. White WB. Blood Pressure load and target organ effects in patients with essential hypertension. J Hypertens, v. 9 (Suppl 8), 1991; 9: S39-41.

36. Staessen J, Fagard R, Thijs L, Amery A et al. A consensus view on the technique of Ambulatory Blood Pressure Monitoring. Hypertension, 1995; 26: 912-18.

37. Consensus on non-invasive Ambulatory Blood Pressure Monitoring. J Hypertens, 1990; 8: 135-40

38. Myers MG, Brian R, Rabki. Canadian Hypertension Society Guidelines for Ambulatory Blood Pressure Monitoring. Am J Hypertens, 1999; 12: 1149-57

39. Nobre F. Análise dos dados obtidos e emissão de laudos. In: Mion Jr D, Nobre F, Monitorização ambulatorial da pressão arterial. 2 ed. São Paulo: Atheneu, 1998.

40. Ayman D, Goldshine AD. Blood pressure determinations by patients with essential hypertension: the difference between clinic and home readings before treatment. Am J Med Sci, 1940; 200: 465-70.

41. Gomes MAM, Pierin A, Segre CA, Mion Jr D. Monitorização residencial da pressão arterial e monitorização ambulatorial da pressão arterial versus medida da pressão arterial no consultório. Arq Bras Cardiol, 1998; 71: 581-5.

42. Padfield PL, Lindsay BA, Mc Laren JA et al. Changing relation between home and clinic blood pressure measurements: do home measurements predict clinic hypertension? Lancet, 1997; 2: 322-4.
43. Bortolotto LA, Henry O, Hanon O et al. Validation of two devices for self-measurement of blood pressure by elderly patients according to the revised British $\mathrm{Hy}$ pertension Society protocol: the Omron HEM-722C and HEM-735C. Blood Pressure Monit, 1999; 4: 21-5.

44. Friedman RH, Kazis LE, Jette A et al. A tele communications system for monitoring and counseling patients with hypertension. Impact on medication adherence and blood pressure control. Am J Hypertens, 1996; 9: 285-92.

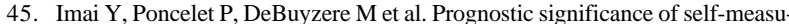
rements of blood pressure. Blood Press Monit, 2000; 5: 137-43.

46. Yarows S. Home blood pressure monitoring in primary care. Blood Pressure Monitoring, 1998; 3: S11-7.

47. Pickering TG, Krakoff L et al. American Society of Hypertension Expert Panel: conclusions and recommendations on the clinical use of home (self) and ambulatory blood pressure monitoring. Am J Hypertens, 1996; 9: 1-11.

48. Vaur L, Dubroca I, Dutry-Aupagne C et al. Superiority of home blood pressure measurements over office measurements for testing antihypertensive drugs. Blood Press Monit, 1998; 3: 107-14.

49. Soghikian K, Casper SM, Fireman BH et al. Home blood pressure in managemen of hypertension. Can Med Assoc J, 1978; 119: 1034-9.

50. Staessen JA, O`Brien ET, Thijs L, Fagard RH. Modern approaches to blood pressure measurements. Occup Eviron Med, 2000; 57: 510-20.

51. Campbell NRC, Abbott D, BASS M et al. Self-measurement of blood pressure: recommendations of the Canadian Coalition for High Blood Pressure Prevention and Control. Canad J Cardiol, 1995; 11: 5-19.

52. Mengden T, Chamontin B, Chau NPet al. User procedure for self-measurements of blood pressure. Blood Press Monit, 2000; 5: 111-29.

53. O'Brien E, Régis DG, Guillaume B et al. Devices and validation. Blood Press Monit, 2000; 5: 93-100.

54. O'Brien E. Replacing the mercury sphygmomanometer. BMJ, 2000; 320: 815-6.

55. Joint National Commitee on Detection, Evaluation and Treatment of High Blood Pressure. The sixth report of the Joint National Commitee on Detection, Evaluation and Treatment of High Blood Pressure (JNC-VI). Arch Intern Med, 1997; 157: 2413-46.

56. O'Brien E, Mee F, Atkins $\mathrm{N}$ et al. Evaluation of three devices for self-measurement of blood pressure according to revised British Hypertension Society Protocol: the Omron HEM-705 CP, Philips HP 5332, and Nissei DS-175. Blood Press Monit, 1996; 1: 55-61.

57. Stergiou GS, Skeva II, Zourbaki AS et al. Self-monitoring of blood pressure a home: how many measurements are needed? J Hypertens, v. 16, p. 725-731, 1998.

58. Stergiou GS,. Zourbaki AS, Skeva II et al. White coat effect detected using self-monitoring of blood pressure at home. Am J Hypertens, 1998; 11: 820-7.

59. Gomes MAM, Pierin A, Mion Jr D et al. Home blood pressure measurement with a automatic device (OMRON IC): acceptance and comparison office blood pressure measurement. Am J Hypertens, 1999; 12.

60. Gerin W, Pickering TG, Holland JK, Alter R. Telephone-linked home blood pressure monitoring may improve management. Am J Hypertens, 1999; 12.

61. Denolle T, Waeber B, Kjeldsen S et al. Self-measurement of blood pressure in clinical trials and therapeutics applications. Blood Press Monit, 2000; 5: 145-9.

62. Tsuji I, Imai Y, Nagai K et al. Proposal of reference values for home blood pressure measurement. Prognostic criteria based on a prospective observation of the general population in Ohasama, Japan. Am J Hypertens, 1997; 10: 409-18.

63. Julius S, Mejia A, Jones K et al. "White coat" versus "sustained" borderline hypertension in Tecumseh, Michigan. Hypertension, 1990; 16: 617-23.

64. Thijs L, Staessen JA, Celis H et al. Reference values for self-recorded blood pressure. A meta-analysis of summary data. Arch Intern Med, 1998; 158: 481-8.

65. WEISSER B, MENGDEN T, DÜSSING R et al. Normal values of blood pressure self-measurement in view of the 1999 World Health Organization - International Society of Hypertension Guidelines. Am J Hypertens, 2000; 13: 340-3.

66. III Consenso Brasileiro de Hipertensão Arterial. III CBH. Rev Bras Clin Ter, 1998; 24: 231-72. 